\title{
SDL SPECIFICATION AND VERIFICATION OF UNIVERSAL PERSONAL COMPUTING WITH OBJECTGEODE
}

\author{
M. Törö, J. Zhu, V. C. M. Leung \\ Dept of Electrical and Computer Engineering \\ The University of British Columbia \\ Vancouver, BC, Canada V6T 1 Z4
}

\begin{abstract}
Support of nomadic computing on the Internet requires the design of new protocols handling issues of user mobility. Our efforts target the universal personal computing (UPC) system to provide a continuous personal computing environment for mobile users. We selected SDL as a specification language for this new service, which allowed us to use Verilog's SDL tool: ObjectGEODE, for design verification. In the paper, we discuss the main principles of UPC, and how the simulator and verifier of ObjectGEODE were used during the stepwise system design. We illustrate the types of errors detected by the verifier, and show that the consistent application of the SDL methodology and the tool has increased our confidence in the correctness of our specification. The specification is the basis of our further work on UPC prototyping.
\end{abstract}

\section{Introduction}

Widespread use of laptop, palm-top computers, PDAs and the possibility of wireless connection to communication and computer networks anticipate the ubiquitous support of nomadic computing on the Internet. One of the main goals of 
our IWIN (Internetworking Wireless Data Networks for Mobile Computing) project involves the design and prototyping of a universal personal computing (UPC) system (Li, Y. and Leung, V.C.M., 1997a, b), which allows nomadic users of the Internet to work in their familiar personal computing environment regardless of their locations, or means of access. We envision that the UPC service will be provided at any location where Internet access is available, so that users everywhere can benefit from it, even during migration, through any available terminals or computers owned by the user or provided by the service provider or a third party on location.

To achieve our goals several new techniques are employed and integrated into our system. We selected SDL (Specification and Description Language) of ITU (ITU Z.100, 1992) and the related methodology to reinforce the design and development process with formalism.

Our design of UPC relies on the following emerging techniques/technologies:

- The Internet Engineering Task Force approved in October 1996 the IP Mobility Support (MIP) protocol (RFC 2002, 1996), which enables terminal mobility on the Internet that is transparent to the TCP level.

- We achieve a homogenous computing environment by applying CORBA (Common Object Request Broker Architecture, 1997) technology in UPC. The IDL (Interface Definition Language) of CORBA provides a common basis to design interchangeable components. CORBA services and facilities are predefined building blocks for new systems.

- Java's (Arnold, K. and Gosling, J., 1997) platform independence allows the portability of components. In the case of the CORBA based UPC, the client objects are brought to the nomadic user's site to deliver the familiar computing environment.

We started our work with the investigation of the IP Mobility Support protocol. We specified it in SDL. This was also an exercise of the specification of mobility with SDL. We reported this work in (Törö, M., 1997).

Currently we are working on the SDL design of the UPC system. We have chosen a stepwise approach: after specifying the basic design, new features are added gradually. After each addition or modification of the design we verify the correctness of the design. Besides, between the major steps we also validate that the new version of our design still satisfies the original requirements. This work essentially differs from the previous one, since it is not based on an existing standard or specification. For the design of the UPC system we apply Verilog's SDL tool: ObjectGEODE (ObjectGEODE, 1997). Beside SDL, ObjectGEODE supports MSC (Message Sequence Chart) of ITU (ITU Z.120, 1992), OMT of (Derr, K.W., 1995), and state and entity relationship diagrams to help the design and documentation process. It provides tools for the entire system life cycle. We present this work in the following sections of the paper.

Before going to the details of our results we give a short summary on SDL and ObjectGEODE, the tool we used in our work. In the third section we discuss the UPC system and its specification in SDL. The fourth section brings up the 
verification results and our experience with the tool. We draw our conclusions in the fifth section.

\section{SDL and SDL tool support}

SDL is an asynchronous extended finite-state machine based language standardized by the ITU for specification and description of communication protocols. It was designed for interactive, distributed, real-time systems.

The most important novelty of the 1992 version of SDL was the introduction of object-oriented methodology for the structural elements of the specification. It also introduced the remote procedure call as a new way of synchronous communications between processes.

There are several SDL based tools mainly utilized in the telecommunication industry. Most of them are in-house products and not commercially available. There are two major commercial SDL tools: SDT by Telelogic (Sweden) and ObjectGEODE by Verilog (France). None of these tools support the full Z.100 standard. Only a third, recently available commercial tool: Cinderella by Cinderella (Denmark) claims the full support of the 1992 and 1996 version of the language. As we mentioned, in our current work we have employed ObjectGEODE.

\section{ObjectGEODE}

ObjectGEODE integrates the SDL related methodology with others such as OMT (Object Modeling Technique) to assist the protocol life cycle from the very early design up to the implementation and conformance testing stages. It offers tools for automatic documentation and version maintenance.

ObjectGEODE provides a graphical user interface for specification using the graphical representation of SDL. From the entered specification executable code is generated for both simulation/verification, and implementation. The simulator has the options of interactive or automatic random simulation. The verifier, which is integrated with the simulator, implements breadth-first, depth-first, or supertrace reachability analysis, and liveness analysis. To verify certain properties one can choose between the construction of observers and the specification of the properties in MSC. Afterwards the verifier automatically checks if there is any inconsistency between the specified property and the system behavior, or verifies the presence of (un)required properties observed by the observer. The verification results are presented in several reports: a summary of the detected problems, number of reached states and fired transitions, is listed after each run. The transition and state coverage reports are presented to the user on demand. During verification problematic scenarios are logged, so that one can replay them to analyze the problem.

MSCs are also used in ObjectGEODE to specify test purposes for conformance test suite generation. For each MSC scenario a test case is generated by finding the appropriate transitions in the reachability tree and generating the alternative transitions and the preambles and postambles. The generated test suite is specified in 
TTCN-MP (Tree and Tabular Combined Notation, Machine Processable format, IS9646 Part 3, 1992)

\section{Universal Personal Computing}

\subsection{Informal description of UPC}

UPC is designed to provide a transparent continuous computing environment for mobile users on the Internet. The key issue of the system is the representation of the user's computing environment and its delivery to the current user location. For economic and performance reasons we also require the system to provide the requested services locally whenever it is possible or desired by the user. Our design includes the case where the terminal or computer through which the user accesses the network is a 'foreign' one, e.g., one leased from a third party at the visited location.

As we mentioned in the introduction the emerging CORBA architecture provides a virtually uniform platform for object-oriented technology. In addition Java provides the portability of the required modules or objects. The CORBA architecture allows us to interpret the user's personal computing environment as the set of objects the user usually uses, which we call the user profile. Since the interface between the client and the server part of an application is described in standard IDL, any other object having the same interface can provide a requested service. This way, certain services may be obtained at the user's actual location through negotiation with the local Trader Service, instead of being delivered remotely from the user's home domain. Only if a requested service could not be obtained at a visited location would the service be invoked remotely.

According to the CORBA architecture, the client part of an application delivers the familiar user interface to the user. Therefore it is enough to migrate or copy only the client part to the user's location. It is possible that the required client part can also be obtained at the visited location, but still the user specific preferences should be considered. We define the user profile as the set of clients and the related environment objects. Java provides the portability of the client objects.

Users are identified in the system by their unique logical user identifier (LUI). They login to the network by providing this LUI and the password.

We define a user agent for each user, located in the user's home domain. It is the object in the system to which the LUI is bound, hence it can be found via the Naming Service. The user agent authenticates the user whenever it is contacted from anywhere on the Internet. The user agent also maintains the user profile and delivers it to the current user location after the user has been authorized by the system. Since a given terminal may not support every feature specified in a user profile, the capabilities of each terminal are described in a terminal profile, which is checked against the user profile and only the supported subset of the user profile is transmitted to the user location. 
Since the user agent is always aware of the availability and the current location of the user, its functions are extended to include forwarding of incoming messages to the user, and acting on the user's behalf when the user is off-line.

To maintain a given user-terminal binding and to provide the personal computing environment at a terminal, we define the user-terminal agent. It is started up after the reception of the user profile, based on the features specified in the profile. The userterminal agent takes care of the user requests to start different services. At such request it either starts the negotiation with the local Trader or obtains the required object according to the user profile. Since after a migration of the mobile user some of the local services may be obsolete, the user-terminal agent maintains also a list of these services. Whenever a new location is reached it automatically initiates a new negotiation with the new local Trader to replace the obsolete services. CORBA calls rely on TCP connections, at which level terminal migration is hidden by the MIP protocol. Only the MIP level is aware of which subnetwork the mobile node is currently attached to, therefore we define a signaling of the motion from the MIP level to the user-terminal agent. It is the user-terminal agent's responsibility to decide whether the migration has resulted in a change of the serving object request broker (ORB) as well.

\subsection{SDL specification of UPC}

At the UPC level we can distinguish three groups of entities as entities at the user's home domain, entities at the visited domain, and entities in the user's terminal. We group these entities in three blocks. Since we require CORBA in all parts of our system, in all of these blocks as a minimum requirement a CORBA Naming Service process needs to be defined. In addition, at the terminal and at the visited domain we define Trader Services. As part of the system initialization procedure the Naming Services exchange their process identifiers and name bindings to become known everywhere in the system.

In CORBA, objects are located on the basis of their object references. For this purpose we used the SDL process identifiers in our specification. Therefore a Naming Service registers the name and process identifier bindings in a given block, and for other Naming Services in the system. The Naming Service process contains two states and two procedures with no states. It accepts five input signals.

The Trader Service, on the other hand, maintains a database of the properties of different available services. At request the Trader Service matches them with the required service properties and returns the appropriate object, which in our case is a process identifier. At the current state, this is only an imaginary process identifier, since we have not yet specified the server objects. However, for the comparison of each of the defined properties we have to specify the appropriate operators. The Trader Service is specified as a one-state process with one procedure. We specify only the look up CORBA method; therefore the process has a single valid input signal.

As we mentioned earlier, SDL is an asynchronous language, and the remote procedure call has been added lately to provide synchronous communications. However, our tool does not support this feature yet. CORBA, on the other hand, 
defines synchronous and one-way method invocation through the static invocation interface, and deferred invocation via the dynamic invocation interface. SDL's asynchronous communication is adequate for the one-way and the deferred invocation. To provide synchronous calls in our UPC specification, whenever a synchronous invocation is assumed, we introduce a blocking state, from which only the inputs received in response to the invocation lead out. Any other input is saved for this state. Since we have not specified the object request brokers separately (the SDL interpreter provides their functions of signal distribution), these blocking states are also guarded with timers from indefinite blocking of the system.

Beside the CORBA components we have the following processes in each block as shown in Figure 1:

System UPC

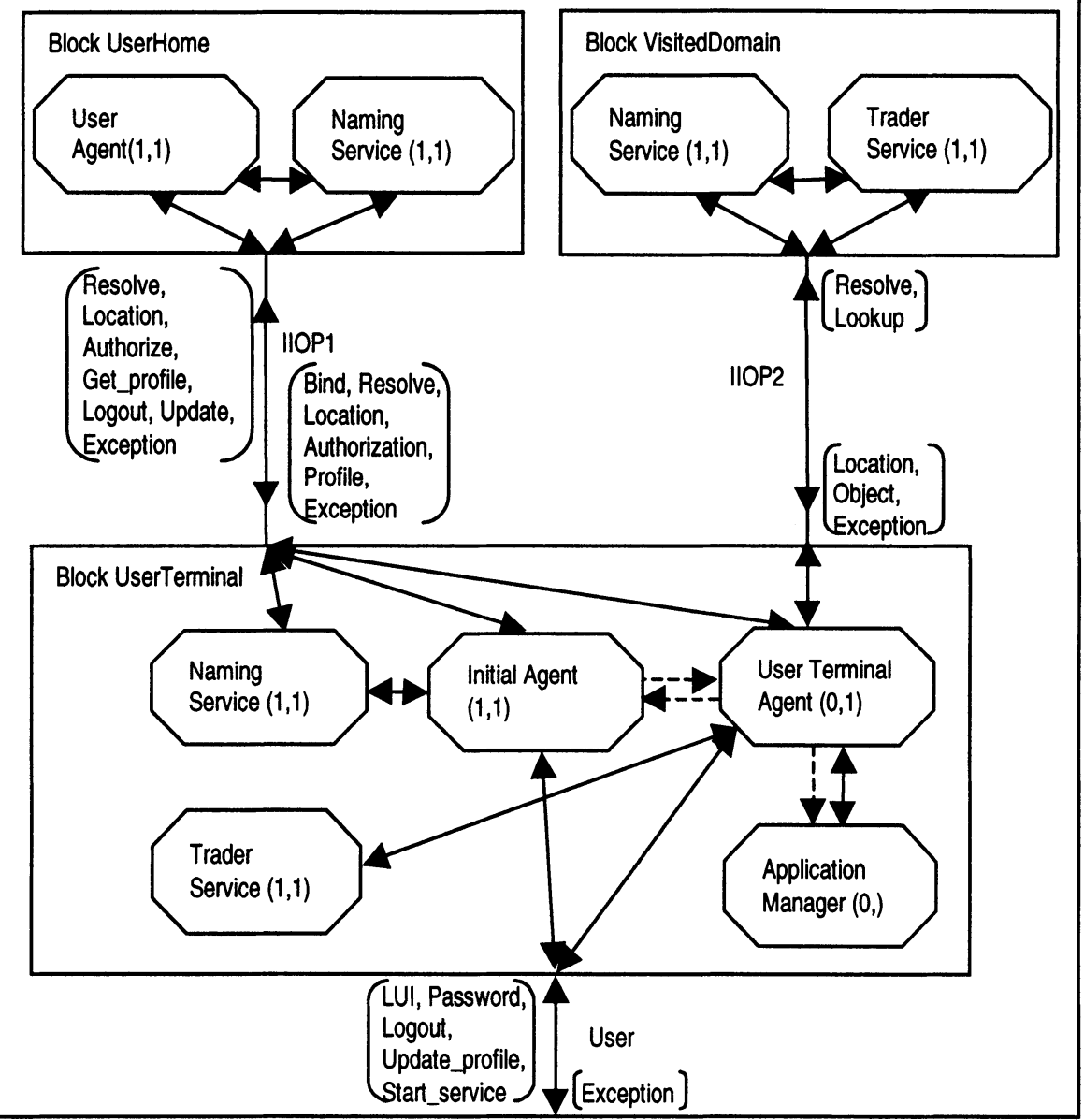

Figure 1 The simplified SDL structure diagram of UPC

- User's home: User Agent process 
- User Terminal: Initial Agent process, User-Terminal Agent process, Application Manager process

- Visited domain: none

The User Agent process is created at the system start and it remains until system termination. Its behavior is straightforward: after initialization, which includes registration of the user's name and process identifier binding with the Naming Service, and initialization of the user profile data structure, the User Agent reaches the off-line state, and remains there until a login is initiated from the User Terminal. The User Agent authenticates the user based on the submitted password. If the authentication is successful, the User Agent moves to the online state, where it stays until a logout signal is received. In the online state the User Agent can send the user profile, update the profile, or deal with exceptions. Accordingly, in the User Agents process, two states and three no-state procedures are specified. It accepts five input signals.

The Initial Agent of the User Terminal accepts the login information: the user's LUI and the password. With the LUI, it finds the User Agent through the Naming Services, and verifies the user provided password with the User Agent. If the authentication is successful, it sends the terminal profile to the User Agent and receives the user profile. Upon the receipt of the user profile, the Initial Agent creates the appropriate User-Terminal Agent for the user, and then terminates. The user login and authorization are realized by seven states and six input signals. The type of the created User-Terminal Agent depends on the characteristics of the terminal and information contained in the user profile.

The User-Terminal Agent represents a given user-terminal binding. It takes care of the actions the user may request: updating the user profile, logout, or starting a service. To start up a service, it first checks in the user profile where to look for the service. If the service should be started at the user's home, the User-Terminal Agent simply creates a new Application Manager and passes the actual parameters required to adjust the service to the user preferences. If the service can be provided locally, it checks first with the User Terminal Trader whether the service is available, and if not it contacts the Trader of the Visited Domain. After receiving the information about a potential server from any of the Traders, this information and the related data from the user profile are passed to a newly created Application Manager. Services provided by the visited domain are restarted any time the user moves to a new domain. The User-Terminal Agent's functionality is specified via four states and seven procedures. Four of the procedures have no states while the rest have 1,2 and 3 states. The process accepts three inputs from the user and five inputs from other processes of the system. The signaling of a change of the terminal location is specified as a spontaneous transition.

The Application Manager administers a given service for the user. It has two states and accepts two inputs sent by the User-Terminal Agent. At service termination it terminates and notifies the User-Terminal Agent about the termination. The termination is specified as a spontaneous transition in the process. If the user requests for an update of the user profile, the User-Terminal Agent collects the 
necessary information from the Application Managers and sends it to the User Agent. At user logout the User-Terminal Agent sends a termination request (one-way request) to the Application Managers, notifies the User Agent about the logout, and after creation of a new Initial Agent instance the User-Terminal Agent also terminates.

Table 1 summarizes the number of process instances, states, input signals and spontaneous transitions in the system.

Table 1 Summary on the UPC system specification

\begin{tabular}{|c|c|c|c|c|c|c|}
\hline Process & $\begin{array}{l}\text { Naming } \\
\text { Service }\end{array}$ & $\begin{array}{l}\text { Trader } \\
\text { Service }\end{array}$ & $\begin{array}{l}\text { User } \\
\text { Agent }\end{array}$ & $\begin{array}{l}\text { Initial } \\
\text { Agent }\end{array}$ & $\begin{array}{l}\text { User } \\
\text { Terminal } \\
\end{array}$ & $\begin{array}{l}\text { Application } \\
\text { A. Manager }\end{array}$ \\
\hline \multicolumn{7}{|l|}{ Number of: } \\
\hline $\begin{array}{l}\text { Process } \\
\text { instances }\end{array}$ & 3 & 2 & 1 & 1 or 0 & 0 or 1 & 0 to 20 \\
\hline States & 2 & 1 & 2 & 7 & 4 & 2 \\
\hline Inputs & 5 & 1 & 5 & 6 & 5 & 2 \\
\hline $\begin{array}{l}\text { Variables } \\
\text { simple } \\
\text { complex }\end{array}$ & $\begin{array}{l}11 \\
1\end{array}$ & $\begin{array}{l}2 \\
2\end{array}$ & $\begin{array}{l}5 \\
3\end{array}$ & $\begin{array}{l}12 \\
2\end{array}$ & $\begin{array}{l}12 \\
4\end{array}$ & $\begin{array}{l}3 \\
1\end{array}$ \\
\hline $\begin{array}{l}\text { States in } \\
\text { procedures }\end{array}$ & 0 & 0 & 0 & 0 & 6 & 0 \\
\hline $\begin{array}{l}\text { Spontaneous } \\
\text { transitions }\end{array}$ & 0 & 0 & 0 & 0 & 1 & 1 \\
\hline
\end{tabular}

Although our design would benefit from the object-oriented extension of SDL (for example for the processes providing CORBA services), we could not use this feature because of restrictions in the tool; i.e., ObjectGEODE does not support context parameters or external synonyms.

\section{Specification and verification of UPC in progress}

\subsection{Specification with the assistance of the verifier}

We started the specification of UPC directly with SDL. We did not use other options offered by ObjectGEODE, since we already had a basic design at the time the tool 
was available to us. We use the stepwise approach to specification, i.e., starting with a basic design, to add new features and changes gradually. After each modification we verify that the system is free from deadlocks or exceptional situations. The following discussion demonstrates this approach.

As the first sketch of the design in SDL, we specified only the main parts of the UPC system and the basic communications between them. SDL allows informal parts in the specification: in tasks and decisions. The ObjectGEODE's simulator allows running such specifications, although it obviously cannot interpret the informal parts. The simulation at this stage helped us in the general understanding of the basic characteristics of the system. The verification could not give any useful results at this stage.

As the next step, we replaced the informal parts related to the user profile, i.e., we worked out a detailed data structure and operations on it. This modification allows us to use the verifier. A typical report is shown in Table 2(a).

The verification summary gives information about the type of executed verification, the number of reached states and transitions covered, the maximum depth reached during the exploration and execution time. The detected errors are reported in three categories: an exception refers to a dynamic error during execution; a deadlock is a system state when no transition can be fired in the system; a stop condition is a condition defined by the user, usually related to a given feature under investigation. Additionally the percentages of covered states and transitions are given. The liveness mode also reports the number of loops found with no success states. This method requires observers to identify the success/error states (ObjectGEODE, 1997).

In the second version of our specification we replaced the signal outputs representing synchronous CORBA calls, with the construction of going into a blocking state and setting a timer. In the blocking state all but the response for the call messages are saved. When we made this change, in one of the processes we forgot to specify the transition for the 'exception' signal. This immediately resulted in the report of Table 2(b) about a deadlock situation in the system.

Note that the deadlock situation was reported only by the breadth-first analysis. Although the depth-first method reported a similar coverage as shown in Table 2(c), it did not detect the deadlock for the same specification.

When we thought that the deadlock was corrected we restarted the verification and received the report of Table 2(d) with a number of exceptions (i.e., dynamic errors) found. Again only the breath-first analysis reported the exceptions; none of the other methods found any error in the specification.

The dynamic errors were the result of only partially correcting the deadlock, by adding the 'exception' input in the blocking 'wait_location' state of the User Terminal Agent, but forgetting the appropriate value assignment to the 'local' variable, which contained the reference (process identifier) to the visited domain's Trader Service. Since the 'exception' signal meant that there was no Trader available at the visited domain, the variable should not be used subsequently for the service lookup call. However, because of the obsolete value, this call was issued, and no recipient was found in the system. 
Table 2 Summary of verification results

\begin{tabular}{|c|c|c|c|c|c|}
\hline & (a) & (b) & (c) & (d) & $(e)$ \\
\hline Method: & supertrace & breadth & depth & breadth & breadth \\
\hline $\begin{array}{l}\text { Number of } \\
\text { states: }\end{array}$ & 162030 & 262142 & 262142 & 262142 & 524286 \\
\hline $\begin{array}{l}\text { Number of } \\
\text { transitions: }\end{array}$ & 433021 & 684944 & 719355 & 723606 & 1566044 \\
\hline $\begin{array}{l}\text { Maximum } \\
\text { depth reached: }\end{array}$ & 30088 & 37 & $\begin{array}{l}1000 \\
\text { (truncated) }\end{array}$ & 35 & 35 \\
\hline $\begin{array}{l}\text { Maximum } \\
\text { breadth reached: }\end{array}$ & $\mathrm{n} / \mathrm{a}$ & 90506 & $\mathrm{n} / \mathrm{a}$ & 86714 & 148866 \\
\hline Duration: & $11 \mathrm{mn} 28 \mathrm{~s}$ & $6 \mathrm{mn} 16 \mathrm{~s}$ & $17 \mathrm{mn} 50 \mathrm{~s}$ & $13 \mathrm{mn} 56 \mathrm{~s}$ & $18 \mathrm{mn} 28 \mathrm{~s}$ \\
\hline $\begin{array}{l}\text { Number of } \\
\text { exceptions: }\end{array}$ & 0 & 0 & 0 & 18991 & 0 \\
\hline $\begin{array}{l}\text { Number of } \\
\text { deadlocks: }\end{array}$ & 0 & 1 & 0 & 0 & 0 \\
\hline $\begin{array}{l}\text { Number of stop } \\
\text { conditions: }\end{array}$ & 0 & 0 & 0 & 0 & 0 \\
\hline $\begin{array}{l}\text { Transitions } \\
\text { coverage rate: } \\
\text { transitions } \\
\text { not covered }\end{array}$ & $\begin{array}{l}46.15 \\
77\end{array}$ & $\begin{array}{l}62.94 \\
53\end{array}$ & $\begin{array}{l}62.94 \\
53\end{array}$ & $\begin{array}{l}59.21 \\
62\end{array}$ & $\begin{array}{l}56.60 \\
69\end{array}$ \\
\hline $\begin{array}{l}\text { States coverage } \\
\text { rate: } \\
\text { states not } \\
\text { covered }\end{array}$ & $\begin{array}{l}76.00 \\
12\end{array}$ & $\begin{array}{l}94.00 \\
3\end{array}$ & $\begin{array}{l}94.00 \\
3\end{array}$ & 88.68 & 88.68 \\
\hline
\end{tabular}

Note that the current version of SDL would not consider this situation an error; however ObjectGEODE reports them at verification and interrupts the simulation in interactive or random mode. To avoid exceptions and the consequent interruption of the simulation in our dynamically changing system configuration, we use timers to delay the process termination whenever we expect such a situation. 
After correction of these errors we ran all verifications again, and no new problem was found. However, the request-response pairs could not be matched in the system. The message identification was lacking. To correct it, we added a sequence number field to the messages, so that an old response could not be accepted any more for a newer request. We also guarded all such calls with timers. After all these changes we ran the verifications again, and this time the verifier reported no errors by any of the methods. We show only the report of the breadth-first analysis in Table 2(e).

A nice feature of ObjectGEODE is that the new verification results update the previous results and so does the simulation. This is important because different methods capture different parts of the system state space. This was the reason why the depth-first method did not find the deadlock, and this is the explanation of the following experiment:

During the verification we tried different parameter settings for the depth-first analysis. Originally we defined the truncation level of the reachability tree at depth 1000 , which normally resulted in a poor state and transition coverage compared to runs of the breadth-first analysis, where the number of explored states and transitions were similar. By defining the truncation level closer $(500,100,50)$ to the reached maximum depth of breadth analysis (35), we got similar results with both methods, since the smaller truncation level forced the verifier at the depth-first analysis to go through the similar part of the reachability tree. The higher numbers of truncation kept the analysis in the left most part of the reachability tree.

Currently we are refining the third version of the UPC specification. To check the consistency of a new version of the specification with an older one, we generated a number of message sequence charts from the older version and ran the new version against them. This serves to verify that a refinement retains the properties of a previous version. For such verification ObjectGEODE generates observers from a MSC document. We found no inconsistency between the different versions of the design so far.

\subsection{State and transition coverage}

We ran the verifications without any limitation set on the exploration size, so all the runs used the maximum memory available, and stopped when they reached this limit. Eventually, as seen in Table 2(e), we reached approximately half a million system states, and one and a half million transitions were investigated. There would be no other way of verifying such a number of states and transitions except with the use of an appropriate tool.

One can see from the report in Table 2, that there were still uncovered states and transitions. To find out more about these we used the state and the transition coverage reports of ObjectGEODE.

The state coverage report lists all the states for all the processes and procedures and for each of them gives the number of hits cumulatively for all instances. Beside the process and procedure state symbols (unlike in the verification results, in the coverage report the data part is not considered) the tool takes into account the process and procedure start symbols as states. The coverage rate is calculated as the 
proportion of states visited at least once to the number of states of the process or procedure. Table 3 presents an excerpt of a state coverage report.

Table 3 Excerpt from state coverage report

states coverage of userhome!namingservice : rate 66.67

start : 1

listen : 72731

wait : 0

states coverage of userhome!namingservice!find : rate 100.00

start : 64512

states coverage of useragent : rate 100.00

start : 1

online : 87694

offline : 34967

states coverage of check_profile : rate 100.00

start : 95

states coverage of update_profile : rate 100.00

start : 87336

states coverage of useragent!create_profile : rate 100.00

start : 29491

From the state coverage list we can easily determine the states which have not been checked during the verification. In this case the unchecked states are the states of the Naming Services, which have been introduced for compatibility with the CORBA standard but are not used by the UPC system. In other words, we have covered all the reachable states in the system; hence there is no need of further verification from this point of view.

The transition coverage report presents the cumulative statistics on transitions (state-input pair) fired in each process and procedure. It also gives the proportion of transitions fired at least once to the number of transitions in the process or procedure. Table 4 shows an example of transition coverage report.

One can see that ObjectGEODE lists as transitions the procedure calls and automatically adds a 'discard' transition for each process or procedure, representing the SDL implicit transition (i.e., an input for which there is no transition specified explicitly so it is discarded in a given state). The tool also adds a time-out counter for each timer of a process. These automatic additions and the timer handling are the features of ObjectGEODE we found arguable.

The simulator and the verifier of ObjectGEODE handle differently the signals entered from the system environment (composing the feed-list) and the internal signals with respect to the time-out signals. If a time-out signal's alternative is from 
the feed-list (i.e., from the environment), the time-out signal is encountered as an alternative, otherwise not. As a result, transitions which are triggered by time-outs and have alternative internal signals are never executed during automatic verification, which in our case includes most of the time-outs in the system. We could check these transitions only by interactive simulation, i.e., by stepping forward the system's timer, so the time-out would happen, and the alternative became available. At this point, when we had to do the job manually, we really appreciated the convenience of the automatic verification for all the other cases.

Table 4 Excerpt from transition coverage report

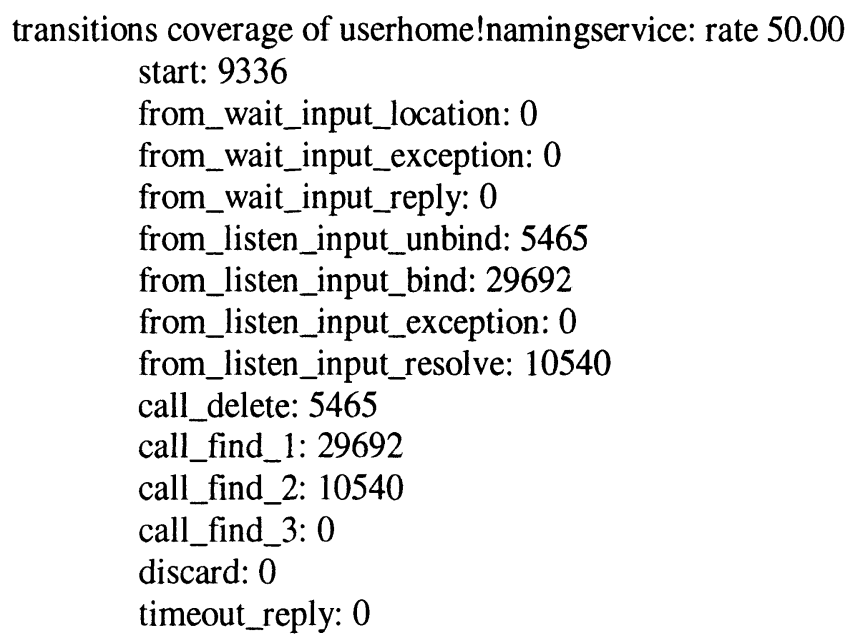

Furthermore, in the current way of interpretation, a time-out would be encountered several times at the calculation of the transition coverage as the report of Table 4 shows. It would be taken into account once when the specification encounters the time-out signal (e.g. from_wait_input_reply), and once at the automatically added 'timeout_reply' transition.

We also debate with the suitability of two other automatically added items on the report, i.e., the introduced 'discard' transition and the procedure calls (e.g. call_find_1). In the case of the discard transition, our problem is that the tool does not check whether or not all of the inputs are handled explicitly by the specification. This addition is justified only when some of the inputs are not handled by the specification. Otherwise this addition falsifies the transition coverage rate. It is further questionable whether it is better to add a 'discard' transition for each state or state-input pair, or one for the whole process. They will give different coverage rates. In any case, the main issue for the designer is to be aware of the fact that a signal has been discarded by the system via an implicit transition. For this purpose any of the solutions is helpful. Regarding the inclusion of the procedure calls in the process transition coverage again the question is whether or not this figure is important for 
the designer. Since the transition coverage is listed for the procedures similarly as for the processes we do not see the need of the inclusion of these calls. In contrary, encountering each of them as a transition corrupt the transition coverage report for the caller process or procedure. In reality these calls might not represent different transitions at all; for example if they were subsequent calls in the same transition. They might not represent different state transitions at all, if the procedures contain no states, or on the opposite, they might hide several transitions if a procedure contains a state with multiple possible inputs.

It is important to be aware of how ObjectGEODE interprets these characteristics, so one can compare the reported results with others calculated by another approach.

In our case after analysing the results and taking into account all these, it can be seen that we have reached the maximum possible transition coverage for the specification. The recalculated state coverage after excluding the start transitions was 96.27. Uncovered states were only those in the Naming Service introduced for compatibility. The transition coverage was 73.11 after excluding the procedure calls, time-out counters and 'discard' transitions where the automaton was fully specified. Uncovered transitions were related to the uncovered states and timers or error handling, which would not happen during automatic verification. They had to be checked manually.

\section{Conclusions}

We have found very useful and effective the application and the assistance of ObjectGEODE in the design of UPC. During this work the static and dynamic checkers of the tool brought our attention to any possible inconsistency in our specification. In many cases these were only warnings, but many times they helped to spot errors, which would be extremely difficult to find without this assistance. Most of the errors were result of inattentiveness. It was trivial to correct them, as we showed in our example, once we knew that there was an error in our specification. However, even at the early stage of the work it was difficult to take into account all possible states and behaviors of our system even with the help of the simulator, because of its distributed and interactive feature. The simulation and the subsequent verification gave us confidence of the correctness and completeness of the specification.

Selecting a standard language like SDL gave us the advantage of:

- The application of the methodology worked out for the language based on the experience of many researchers and technicians around the world, and

- A powerful tool, ObjectGEODE, which was introduced to the market several years ago and is gradually finding its way to acceptance by the industry.

In spite of some short-comings, ObjectGEODE's capabilities are overwhelming in comparison with the in house tools we used earlier. We are convinced that to design a complex system, which is typical for modern communication systems, such design help and assistance as provided by ObjectGEODE are imperative from the 
very beginning of the work. ObjectGEODE is also easy to learn and use. There was some awkwardness in the earlier version of the tool, but most of it was corrected in the newversion 3.2. The new HTML based manual is more convenient, though addition of some more links would be a great help for the user.

We are continuing our work on the UPC system with further refinements of the specification. Some of the features we plan to add include allowing multiple userterminal bindings, specification of the type hierarchy of the user-terminal agents, and refinement of the application manager.

Beside the specification, we are also working on prototyping the system, based on the specification. This work was started before the final specification, since the prototype will not implement all the options specified in the final version of the design. At the same time, practical implementation issues bring up new questions which help us in the refinement of the specification. For prototyping we could use the $C$ code generated by the design tool. Unfortunately, as we mentioned earlier, many of the system components require Java implementation for portability, which is not yet supported in ObjectGEODE or any other SDL based tool we know.

\section{Acknowledgment}

This work was supported by a grant from Motorola Wireless Data Systems Division, Richmond, B.C., Canada.

\section{References}

1. Arnold, K. and Gosling, J. (1997) The Java programming language, AddisonWesley.

2. CORBA (1997) Common Object Request Broker Architecture documentation, Object Management Group, http://www.omg.org

3. Derr, K.W.(1995) Applying OMT: A Practical Step-By-Step Guide to Using the Object Modeling Technique, SIGS Books \& Multimedia.

4. IS-9646 Part 3 (1992) Tree and Tabular Combined Notation

5. ITU Z.100 (1992) Specification and Description Language (SDL).

6. ITU Z.120 (1992) Message Sequence Chart (MSC).

7. Li, Y. and Leung, V.C.M.(1997a) Supporting personal mobility for nomadic computing over Internet, ACM Mobile Computing and Communications Review, Vol.1, No.1, 22-31.

8. Li, Y. and Leung, V.C.M.(1997b) Protocol architecture for universal personal computing, IEEE Journal on Selected Areas in Communication, October 1997

9. ObjectGEODE (1997) documentation, Verilog, Inc.

10. RFC 2002 (1996) IP Mobility Support, Internet Engineering Task Force.

11. Törö, M. (1997) Simulation of IP Mobility Support: An experiment in mobile protocol specification with SDL, in SDL, MSC and Trends Proceedings of the Eighth SDL Forum (ed. A. Cavalli and A. Sarma), Elsevier Science. 


\section{BIOGRAPHY}

Mária Törö received her Ph.D. in Computer Science from the Technical University of Budapest in 1995. Her main field of expertise is formal description techniques and their application for specification and conformance testing of communication protocols. Currently she works on the SDL design and specification of the UPC system in the IWIN project at the University of British Columbia. Her previous experience includes formal specification, analysis and testing of the mobile IP, X.25 and other protocols, and development of tools for protocol engineering at UBC and at the Research Institute for Measurement and Computing Techniques (Hungary).

Jinsong Zhu received his B.Sc. and M.Sc. in Computer Science from Tsinghua University, China in 1985 and 1987 respectively. He was a faculty member at Tsinghua University from 1987 to 1991 . From 1995 to 1996 , he was a Senior Software Engineer at Infonet Software Solutions, Inc., Canada. He is currently working towards his Ph.D. degree at the Computer Science Department of the University of British Columbia. His research interests include protocol testing and verification, high speed networks, mobile computing and distributed object computing technology.

Victor C. M. Leung received the B.A.Sc. and Ph.D. degrees in Electrical Engineering from the University of British Columbia (U.B.C.) in 1977 and 1981, respectively. From 1981 to 1987 , he was a Member of Technical Staff at MPR Teltech Ltd., Burnaby, B.C., specializing in the design and analysis of satellite systems. He lectured in the Department of Electronics at the Chinese University of Hong Kong in 1988. Since 1989, he has been a faculty member with the Department of Electrical and Computer Engineering at U.B.C., where he is currently an Associate Professor. His research interests are in telecommunications and computer networking. Dr. Leung is a senior member of the IEEE, and a voting member of the ACM. 